\title{
A New Sympathovagal Balance Index from Electrodermal Activity and Instantaneous Vagal Dynamics: A Preliminary Cold Pressor Study
}

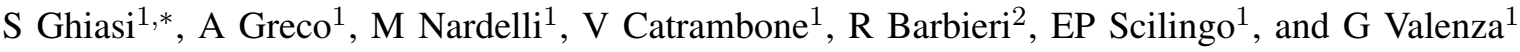

\begin{abstract}
Sympathovagal balance, an autonomic index resulting from the sympathetic and parasympathetic influences on cardiovascular control, has been extensively used in the research practice. The current assessment is based on analyzing Heart Rate Variability (HRV) series in the frequency domain by regarding the ratio between the low and high frequency components (LF/HF). Nevertheless, LF and HF powers are known to be both influenced by vagal activity which strongly bias the accuracy of this method. To this extent, in this study we combine time-varying estimates from electrodermal activity (EDA) and HRV to propose a novel index of sympathovagal balance. Particularly, sympathetic activity is estimated from the EDA power calculated within the $0.045-0.25 \mathrm{~Hz}$ bandwidth $\left(E D A_{S y m p}\right)$, whereas parasympathetic dynamics is measured instantaneously through a point-process modeling framework devised for heartbeat dynamics $\left(H F_{p p}\right)$. We test our new index $\mathcal{S}=E D A_{S y m p} / H F_{p p}$ on data from 22 healthy subjects (7 females and 15 males) undergoing a 3 minutes gold-standard protocol for sympathetic elicitation as the cold-pressor test (CPT). Results show that the activation of the proposed sympathovagal tone is consistent with CPT elicitation and is associated with a significantly higher statistical discriminant power than the standard LF/HF ratio, also revealing different dynamics between female and male subjects.
\end{abstract}

\section{INTRODUCTION}

Autonomic Nervous System (ANS) dynamics, resulting from sympathetic and parasympathetic outflow, has a fundamental role in the regulation of cardiovascular homeostasis [1]. Hence, Heart Rate Variability (HRV) analysis has been widely used for the non-invasive assessment of ANS activity on heartbeat control [1].

Previous research has suggested that spectral analysis of HRV series can provide quantitative markers of sympathetic and parasympathetic outflow of the ANS [1]. Particularly, while it has been established that the power of the high frequency (HF) components of HRV reliably characterizes parasympathetic activity, the interpretation of the physiological correlates behind cardiovascular oscillations in the low frequency (LF) band has been challenged [2]. Consequently, the LF power is now regarded as a measure of both sympathetic and vagal tone, leading to ambiguities and possible inconsistent conclusions on the use of the ratio between $\mathrm{LF}$ and HF power (LF/HF), which is often employed as an marker of sympathovagal balance though [2].

An alternative way to assess the sympathetic function of the ANS is to analyze the electrodermal activity (EDA) signal

\footnotetext{
${ }^{1}$ Computational Physiology and Biomedical Instruments group, Department of Information Engineering \& Bioengineering and Robotics Research Centre E. Piaggio, School of Engineering, University of Pisa, Pisa, Italy

${ }^{2}$ Department of Electronics, Informatics and Bioengineering, Politecnico di Milano, Milano, Italy.

*E-mail: shadi.ghiasi@centropiaggio.unipi.it
}

[3]-[5]. EDA refers to autonomic changes in the electrical conductance of the skin. During sympathetic activation, the sudomotor glands produce sweat which results in alternations to the skin conductivity. Therefore, EDA has been considered to embed signs of purely sympathetic autonomic activity [6]. To this extent, the EDA power calculated within a specific frequency band has been proposed as a sensitive marker of sympathetic dynamics, namely EDA $A_{\text {Symp }}[6]$.

In this study, we test the ability of combined HRV and EDA measures in characterizing sympathovagal balance. Specifically, we study the ratio between $E D A_{S y m p}$ from EDA vs. HF power from HRV in a gold-standard experiment evoking sympathetic activation known as the cold-pressor test (CPT) [7]. In fact, non-baroreflex-mediated sympathetic neural control in humans has been evaluated using CPT in both clinical and experimental settings [8]-[10]. Exemplarily, heart rate and blood pressure responses to the CPT were assessed in male and female healthy subjects [11], [12], as well as hypertension [13] and depression [14].

From a methodological viewpoint, we tackle the nonstationarity associated with EDA and HRV series and estimate time-frequency EDA representation using standard short-time Fourier transform, while HRV-HF power is derived from a point-process modeling devised for heartbeat dynamics [15]. This choice allows for the instantaneous, fully parametric estimation of the HF power (hereinafter $H F_{p p}$ ), while obtaining goodness of fit measures with no need for a preliminary interpolation of the RR interval. This methodological choice, therefore, allows us to properly combine $E D A_{\text {Symp }}$ and $H F_{p p}$ estimates, which otherwise might be biased by the intrinsic uneven nature of samples corresponding to the heartbeat timing.

We estimate the proposed $E D A_{S y m p} / H F_{p p}$ index of sympathovagal balance in data gathered from 22 healthy volunteers undergoing resting state and CPT elicitation, and test its performance in terms of statistical significance with respect to traditional $L F / H F$ ratio as well as other standard HRV and EDA indices defined in the time and frequency domains. Gender differences and similarities are also discussed.

\section{Materials And Methods}

\section{A. Subjects Recruitment, Experimental protocol and Acqui- sition set-up}

Twenty-two right handed students (15 men and 7 females) of ages between 20 and 35 from University of Pisa participated in this experiment. The experimental procedures were approved by the local ethical committee. The experiment was 
performed in a quiet dark room. Participants did not have any history of neurological and cardiovascular diseases, as well as alcoholic or smoking habits. Subjects were asked to avoid coffee and alcohol and strenuous exercise 2 hours before laboratory visit. At first the subjects signed the informed consent. In order to ensure the hemodynamic stabilization, before the experiment, participants were asked to sit in a comfortable chair while watching a black screen for the period of 4 minutes. For the cold pressor test, subjects were asked to submerge their left hand up to wrist into a tank filled of ice and water with the temperature of 0-4 degrees centigrade for a period of 3 minutes. This choice of CPT duration is consistent with the average pain threshold of healthy subjects [16]. During the recovery phase after the CPT, they were asked to remove their hand from the water, and put it on the towel over the table and relax for 4 minutes. The ECG and EDA signals were acquired using the BIOPAC MP35 device with a sampling rate of $500 \mathrm{~Hz}$. From the ECG, the R-peaks were identified through the well-known Pan-Tompkins algorithm in order to obtain the RR interval series. Physiological and algorithmic artefacts were corrected through a local log-likelihood point-process statistics devised for cardiac dynamics.

\section{B. EDA Analysis and Sympathetic Activity Estimates}

EDA signals were downsampled to $50 \mathrm{~Hz}$ and normalized by applying z-score calculation. Then, a 2th order Butterworth bandpass filter (0.01-0.5 Hz) was employed to remove any very low frequency trend. The time-frequency representation of EDA was calculated through the standard shorttime Fourier transform approach with Welch periodograms. A Blackman window of 60s (overlap of 59s) was employed to divide the signal into segments and obtain the timefrequency representation. Such a time-frequency plane is then integrated within the $(0.045-0.25 \mathrm{~Hz})$ band in order to estimate the sympathetic autonomic activity $E D A_{S y m p}(k)$ where $k$ are discrete time samples within a time window $(0, T]$ (further details in [6]).

\section{Point Process theory and Instantaneous Estimates of Vagal Activity}

The Point process theory is a powerful statistical tool which enables to describe the probabilistic structure of heartbeat generation and obtain instantaneous HRV estimates defined in the time and frequency domains. Within the point process framework, the cardiovascular control dynamics is in fact instantaneously characterized by inverse Gaussian probability density functions defined as:

$$
\begin{aligned}
& f\left(t \mid \mathcal{H}_{t}, \xi(t)\right)=\left[\frac{\xi_{0}(t)}{2 \pi\left(t-u_{j}\right)^{3}}\right]^{\frac{1}{2}} \\
& \exp \left\{-\frac{1}{2} \frac{\xi_{0}(t)\left[t-u_{j}-\mu_{R R}\left(t, \mathcal{H}_{t}, \xi(t)\right)\right]^{2}}{\mu_{R R}\left(t, \mathcal{H}_{t}, \xi(t)\right)^{2}\left(t-u_{j}\right)}\right\}
\end{aligned}
$$

where:
- $t$ the continuous time

- $j$ the index of the previous R-wave event occurred before time $t$

- $\mathcal{H}_{t}=\left(u_{j}, R R_{j}, R R_{j-1}, \ldots, R R_{j-M+1}\right)$ the history of previous heartbeat events with $\left\{u_{j}\right\}_{j=1}^{J}$ as successive R-wave event times extracted from the ECG for $(0, T]$

- $R R_{j}=u_{j}-u_{j-1}>0$

- $\xi(t)$ is the vector of the time-varying parameters

- $\mu_{R R}\left(t, \mathcal{H}_{t}, \xi(t)\right)$ the first-order moment (mean) of $f\left(t \mid \mathcal{H}_{t}, \xi(t)\right)$

- $\xi_{0}>0$ the shape parameter of $f\left(t \mid \mathcal{H}_{t}, \xi(t)\right)$

In this preliminary study we follow the simpler non trivial linear autoregressive model of $\mu_{R R}\left(t, \mathcal{H}_{t}, \xi(t)\right)$ as follows:

$$
\mu_{R R}\left(t, \mathcal{H}_{t}, \xi(t)\right)=\gamma_{0}+\sum_{i=1}^{p} \gamma_{1}(i, t) R R_{j-i}
$$

with second-order moment $\sigma_{R R}^{2}\left(t, \mathcal{H}_{t}, \xi(t)\right)$ defined as:

$$
\sigma_{R R}^{2}\left(t, \mathcal{H}_{t}, \xi(t)\right)=\mu_{R R}^{3}(t) / \xi_{0}(t) .
$$

Here, the estimation of $\xi(t)$ was performed through a local maximum likelihood procedure (details in [17]).

Instantaneous estimates in the frequency domain can be directly derived from the instantaneous power spectra:

$$
\mathcal{Q}(f, t)=\sigma_{\mathrm{RR}}^{2}\left(t, \mathcal{H}_{t}, \xi(t)\right) H_{1}(f, t) H_{1}(-f, t)
$$

where $H_{1}$ is the Fourier transform of the $\gamma_{1}$ terms. Then, $\mathcal{Q}(f, t)$ can be integrated in the low frequency $\left(\mathrm{LF}_{\mathrm{pp}}=0.04\right.$ $0.15 \mathrm{~Hz})$, and high frequency $\left(\mathrm{HF}_{\mathrm{pp}}=0.14-0.45 \mathrm{~Hz}\right)$ bands, also to obtain the standard instantaneous sympathovagal balance $(L F / H F)$. Note that $H F_{p p}(t)$ indicates instantaneous estimates of vagal activity.

\section{The proposed index of Sympathovagal balance}

We take advantage of the aforementioned sympathetic autonomic activity $E D A_{S y m p}(k)$ and instantaneous vagal activity $H F_{p p}(t)$ to define a new index of sympathovagal balance as follows:

$$
\mathcal{S} \mathcal{V}(k)=\frac{E D A_{S y m p}(k)}{\left.H F_{p p}(t)\right|_{t=k}}
$$

The proposed $\mathcal{S}$ index is tested against the standard $L F / H F$ ratio, as well as other autonomic outflow indices derived from instantaneous $\mathrm{HRV}$ and time-varying EDA analyses.

\section{EXPERIMENTAL RESULTS AND DISCUSSION}

Group-wise statistics concerned the difference between the resting state and CPT. Aiming to investigate the primary response to sympathetic elicitation, in this preliminary endeavour the time-varying dynamics of the autonomic features was condensed as the median value along the last $30 \mathrm{~s}$ of rest, as well as the first 30s of CPT. Significant differences between sessions were assessed for each feature through pvalues gathered from non-parametric Wilcoxon signed rank 
tests for paired data, with null-hypothesis of equality of population medians between the resting and CPT conditions.

Experimental results gathered from EDA and instantaneous HRV analysis, namely EDA $A_{S y m p}, \mu_{R R}, \sigma_{R R}^{2}, \mathrm{LF}$, $\mathrm{HF}$, and $\mathrm{LF} / \mathrm{HF}$, as well as from the proposed sympathovagal index $\mathcal{S} \mathcal{V}$ are shown in Table I. Taking into account the non-Gaussian distribution of some data samples, the Table values are expressed as $\operatorname{Median} \pm 1.4826 \operatorname{MAD}(X) / \sqrt{n}$, where $\operatorname{MAD}(X)=\operatorname{Median}(|X-\operatorname{Median}(X)|))$, with $X$ as the defined index and $n$ is the number of subjects in group. Note that, in case of Gaussian distribution, $\sigma(X)=$ 1.4826MAD $(X)$ with $\sigma$ as the sample standard deviation. Group-wise dynamical trackings of the EDA $A_{S y m p}$ and instantaneous HRV estimates, as well as the proposed $\mathcal{S V}$ are shown in Figure 1.

In agreement with previous studies [6], the EDA $A_{S y m p}$ showed significant increases during CPT with respect to resting state. Maximal EDA $A_{S y m p}$ is in about 30s from the beginning of CPT. As expected, a significant decrease in $\mu_{R R}$ during CPT was also observed. The proposed $\mathcal{S}$ showed significant increases during CPT, with local maxima at about $25 \mathrm{~s}$ and $60 \mathrm{~s}$ after the beginning of the prolonged sympathetic elicitation and a decay lasting for about 90s. At a speculation level, these can be linked to the sympathetic oscillations with lower frequency of $1 / 25 \mathrm{~s}=0.04 \mathrm{~Hz}$, which are known to be influenced by the baroreflex [1]. All other HRV-derived features, including standard LF/HF ratio, were associated with non-significant $\mathrm{p}$-values $>0.05$ for the rest vs. CPT comparison.

TABLE I

GROUP-Wise EDA AND HRV STATISTICS FROM ALl SubJeCtS

\begin{tabular}{llll}
\hline & Rest (30s) & CPT (30s) & p-value \\
\hline$E D A_{\text {Symp }}$ & $0.53 \pm 0.08$ & $1.20 \pm 0.21$ & $\mathbf{0 . 0 1 0 8}$ \\
\hline$\mu_{R R}[m s]$ & $854.42 \pm 30.37$ & $780.81 \pm 29.68$ & $\mathbf{0 . 0 0 0 2}$ \\
\hline$\sigma_{R R}^{2}\left[m s^{2}\right]$ & $1007.04 \pm 258.69$ & $876.64 \pm 220.03$ & 0.445 \\
\hline$L F_{p p}\left[\mathrm{~ms}^{2}\right]$ & $1117.72 \pm 261.47$ & $1246.66 \pm 203.14$ & 0.189 \\
\hline$H F_{p p}\left[\mathrm{~ms}^{2}\right]$ & $672.26 \pm 117.72$ & $466.80 \pm 124.72$ & 0.094 \\
\hline $\mathrm{LF} / \mathrm{HF}$ & $2.14 \pm 0.50$ & $2.03 \pm 0.49$ & 0.858 \\
\hline $\mathcal{S} \mathcal{V}$ & $0.001 \pm 0.0002$ & $0.003 \pm 0.0006$ & $\mathbf{0 . 0 0 7 4}$ \\
\hline
\end{tabular}

Aiming to study possible gender differences, we split the the dataset considering statistics from males and females only. Experimental results from male and female subjects are shown in Tables II and III, respectively, with related instantaneous trackings shown in Figures 2 and 3.

TABLE II

GROUP-Wise EDA AND HRV STATISTICS FROM MALE SubJeCtS

\begin{tabular}{llll}
\hline & Rest (30s) & CP (30s) & p-value \\
\hline$E D A_{S y m p}$ & $0.53 \pm 0.08$ & $0.88 \pm 0.16$ & $\mathbf{0 . 0 1 7}$ \\
\hline$\mu_{R R}[m s]$ & $882.62 \pm 31.31$ & $781.002 \pm 38.89$ & $\mathbf{0 . 0 0 0 9}$ \\
\hline$\sigma_{R R}^{2}\left[m s^{2}\right]$ & $1436.66 \pm 379.63$ & $938.82 \pm 409.92$ & 0.770 \\
\hline$L F_{p p}\left[\mathrm{~ms}^{2}\right]$ & $1959.46 \pm 562.23$ & $1690.21 \pm 294.76$ & 0.110 \\
\hline$H F_{p p}\left[\mathrm{~ms}^{2}\right]$ & $589.54 \pm 141.27$ & $447.09 \pm 136.74$ & 0.360 \\
\hline $\mathrm{LF} / \mathrm{HF}$ & $3.22 \pm 0.98$ & $3.13 \pm 0.93$ & 0.770 \\
\hline $\mathcal{S V}$ & $0.001 \pm 0.0002$ & $0.003 \pm 0.0007$ & $\mathbf{0 . 0 1 0}$ \\
\hline
\end{tabular}

Likewise for the results gathered from the whole group, considering the male sample, EDA $A_{S y m p}, \mu_{R R}$, and $\mathcal{S} \mathcal{V}$

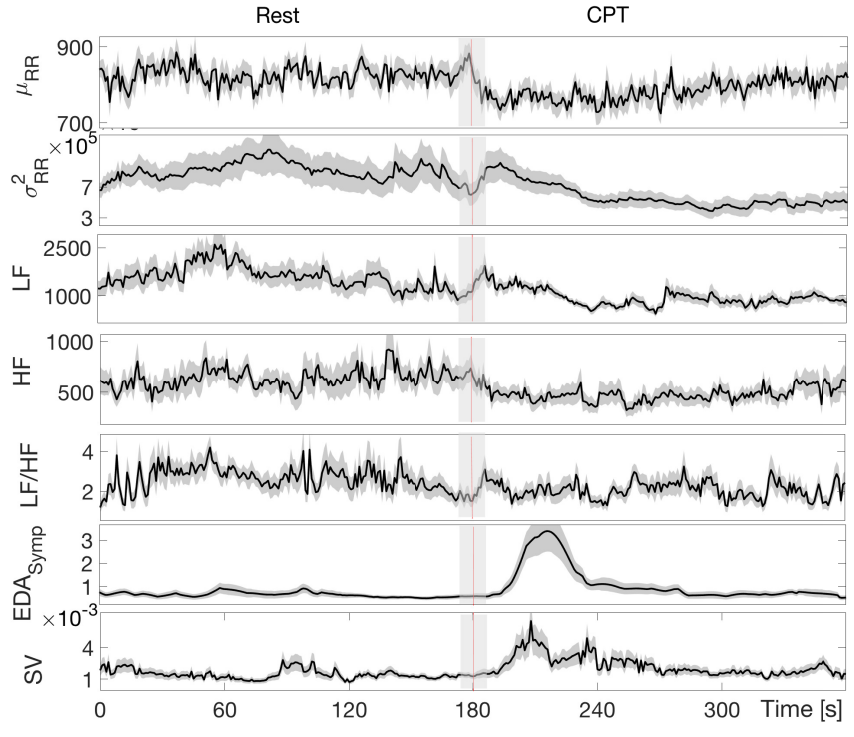

Fig. 1. Instantaneous heartbeat and EDA statistics averaged among all subjects. From the top, the instantaneous $\mu_{R R}, \sigma_{R R}^{2}, \mathrm{LF}, \mathrm{HF}, \mathrm{LF} / \mathrm{HF}$, as well as $E D A_{S y m p}$ and the proposed $\mathcal{S} \mathcal{V}$ are shown. Continuous black lines indicate the median value among all subjects, whereas the superimposed grey areas indicate the MAD. The 10 s grey rectangle at 180 s indicates the transition between rest and CPT, which is marker with a vertical red line.

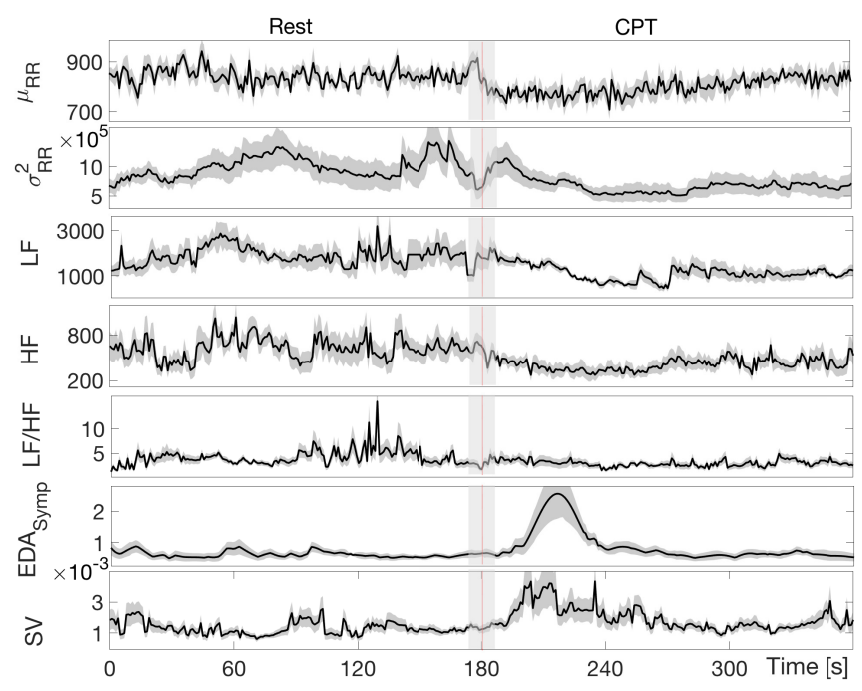

Fig. 2. Instantaneous heartbeat and EDA statistics averaged among male subjects. From the top, the instantaneous $\mu_{R R}, \sigma_{R R}^{2}, \mathrm{LF}, \mathrm{HF}, \mathrm{LF} / \mathrm{HF}$, as well as $E D A_{S y m p}$ and the proposed $\mathcal{S} \mathcal{V}$ are shown. Continuous black lines indicate the median value among all subjects, whereas the superimposed grey areas indicate the MAD. The 10 s grey rectangle at 180 s indicates the transition between rest and CPT, which is marker with a vertical red line.

showed significant differences during CPT with respect to resting state. Note that the proposed $\mathcal{S} \mathcal{V}$ was associated with the lower p-value. Concerning the feature dynamics, maximal EDA $A_{S y m p}$ was in about 30 s from the beginning of CPT, whereas local maximum of $\mathcal{S} \mathcal{V}$ was about 25 s after the beginning of CPT.

No feature showed statistical differences between rest and CTP in the female dataset. However, non-significant p-values may be due to the fact that calculations were performed on a small sample size ( 7 subjects only). On the other 


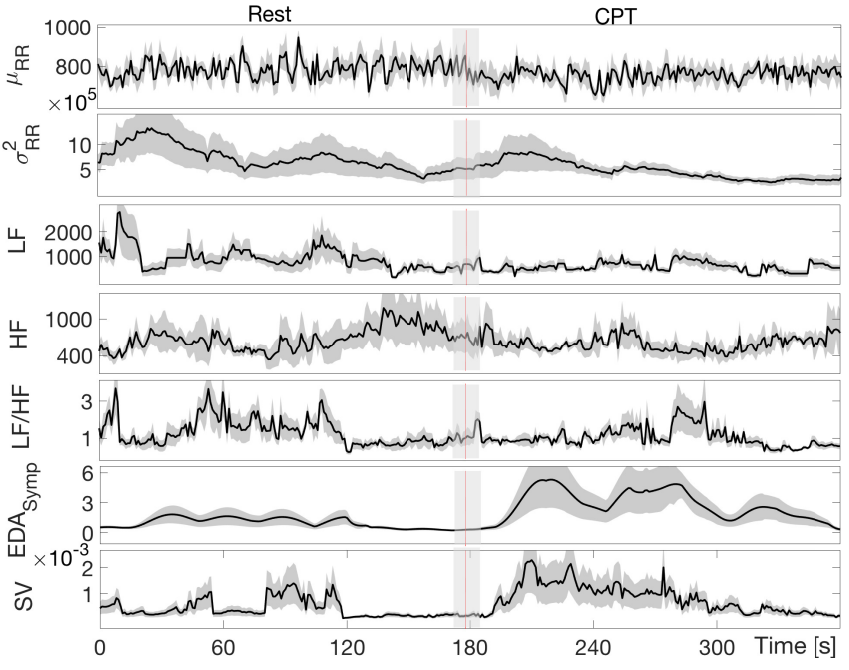

Fig. 3. Instantaneous heartbeat and EDA statistics averaged among female subjects. From the top, the instantaneous $\mu_{R R}, \sigma_{R R}^{2}, \mathrm{LF}, \mathrm{HF}, \mathrm{LF} / \mathrm{HF}$, as well as $E D A_{S y m p}$ and the proposed $\mathcal{S} \mathcal{V}$ are shown. Continuous black lines indicate the median value among all subjects, whereas the superimposed grey areas indicate the MAD. The 10 s grey rectangle at 180 s indicates the transition between rest and CPT, which is marker with a vertical red line.

TABLE III

GROUP-WISE EDA AND HRV STATISTICS FRom Female SubJects

\begin{tabular}{llll}
\hline & Rest (30s) & CPT (30s) & p-value \\
\hline$E D A_{S y m p}$ & $0.32 \pm 0.12$ & $1.62 \pm 0.73$ & 0.31 \\
\hline$\mu_{R R}[m s]$ & $804.61 \pm 69.74$ & $753.52 \pm 46.28$ & 0.09 \\
\hline$\sigma_{R R}^{2}\left[m s^{2}\right]$ & $426.10 \pm 174.02$ & $798.40 \pm 208.63$ & 0.31 \\
\hline$L F_{p p}\left[\mathrm{~ms}^{2}\right]$ & $489.66 \pm 179.06$ & $446.33 \pm 172.51$ & 0.99 \\
\hline$H F_{p p}\left[\mathrm{~ms}^{2}\right]$ & $841.23 \pm 324.13$ & $586.67 \pm 288.64$ & 0.12 \\
\hline $\mathrm{LF} / \mathrm{HF}$ & $0.84 \pm 0.34$ & $0.81 \pm 0.30$ & 0.99 \\
\hline $\mathcal{S V}$ & $0.002 \pm 0.001$ & $0.01 \pm 0.007$ & 0.24 \\
\hline
\end{tabular}

hand, considering feature trends in Figure 3, quite consistent decreasing of HF dynamics, as well as increasing $E D A_{S y m p}$ and $\mathcal{S}$ among subjects can be observed. Note that local maxima in EDA $A_{\text {Symp }}$ are at 30 s and 90 s, whereas $\mathcal{S V}$ showed a peak at $25 \mathrm{~s}$ and a slow decreasing trend lasting 90s (therefore consistently with the trends in Figure 1).

\section{CONCLUSION}

In conclusion, aiming to find a more sensitive marker of sympathetic/parasympathetic balancing effect than the standard $L F / H F$ ratio, we proposed a new index of symapthovagal balance derived from time-varying EDA and HRV estimates. The $L F / H F$ ratio, in fact, is strongly biased by the fact that the $L F$ power of HRV cannot be considered as a reliable marker of purely sympathetic activation [2]. Specifically, we combined sympathetic estimates from EDA according to the so-called EDA $A_{S y m p}$ definition [6] and instantaneous estimates vagal dynamics from point process modeling of heartbeat dynamics [17]. While discerning between resting state and prolonged sympathetic elicitation induced by cold-pressor (CPT), the proposed sympathovagal index was associated with the highest statistical significance (i.e., lowest p-value) than the linked $E D A_{\text {Symp }}$ and $H F_{p p}$ indices, as well as the LF/HF ratio. The time-varying dy- namics of $\mathcal{S} \mathcal{V}$ is also consistent with the current knowledge on sympathetic and parasympathetic outflow dynamics and associated time constants (see Figure 1). Concerning the gender, significant differences were found in $E D A_{S y m p}$ dynamics between males and females. In this regards, however, the different sample size between the female and male datasets is a limitation of this study. Nevertheless, although at a speculation level, consistent feature trends and reduced inter-subject variability were observed for group-wise estimates gathered from the female sample (see Figure 3). Future works will be directed in increasing the sample size, while studying possible alternative rules for combining EDA and heartbeat dynamics maybe accounting for the associated physiological nonlinear dynamics.

\section{REFERENCES}

[1] A. J. Camm et al., "Heart rate variability. standards of measurement, physiological interpretation, and clinical use," European heart journal, vol. 17 , no. 3, pp. 354-381, 1996.

[2] G. A. Reyes del Paso et al., "The utility of low frequency heart rate variability as an index of sympathetic cardiac tone: a review with emphasis on a reanalysis of previous studies," Psychophysiology, vol. 50, no. 5, pp. 477-487, 2013.

[3] H. D. Critchley, "Electrodermal responses: what happens in the brain," The Neuroscientist, vol. 8, no. 2, pp. 132-142, 2002.

[4] W. Boucsein, Electrodermal activity. Springer Science \& Business Media, 2012.

[5] A. Greco, G. Valenza, M. Nardelli, M. Bianchi, L. Citi, and E. P. Scilingo, "Force-velocity assessment of caress-like stimuli through the electrodermal activity processing: Advantages of a convex optimization approach," IEEE Transactions on Human-Machine Systems, vol. 47, no. 1, pp. 91-100, 2016

[6] H. F. Posada-Quintero et al., "Power spectral density analysis of electrodermal activity for sympathetic function assessment," Annals of biomedical engineering, vol. 44, no. 10, pp. 3124-3135, 2016.

[7] J. J. McGinley and B. H. Friedman, "Autonomic responses to lateralized cold pressor and facial cooling tasks," Psychophysiology, vol. 52, no. 3, pp. 416-424, 2015.

[8] M. Muzi et al., "Clonidine reduces sympathetic activity but maintains baroreflex responses in normotensive humans." Anesthesiology, vol. 77, no. 5, pp. 864-871, 1992.

[9] L. Mourot, M. Bouhaddi, and J. Regnard, "Effects of the cold pressor test on cardiac autonomic control in normal subjects," Physiological research, vol. 58, no. 1, p. 83, 2009.

[10] S. Betti et al., "Evaluation of an integrated system of wearable physiological sensors for stress monitoring in working environments by using biological markers," IEEE Transactions on Biomedical Engineering, 2017.

[11] J. L. Wirch et al., "Cold pressor test protocol to evaluate cardiac autonomic function," Applied physiology, nutrition, and metabolism, vol. 31, no. 3, pp. 235-243, 2006.

[12] R.-C. Peng et al., "Time-frequency analysis of heart rate variability during the cold pressor test using a time-varying autoregressive model," Physiological measurement, vol. 36, no. 3, p. 441, 2015.

[13] M. Menkes et al., "Cardiovascular reactivity to the cold pressor test as a predictor of hypertension." Hypertension, vol. 14, no. 5, pp. 524 $530,1989$.

[14] J. W. Hughes and C. M. Stoney, "Depressed mood is related to high-frequency heart rate variability during stressors," Psychosomatic medicine, vol. 62, no. 6, pp. 796-803, 2000.

[15] G. Valenza, L. Citi, A. Lanatá, E. P. Scilingo, and R. Barbieri, "Revealing real-time emotional responses: a personalized assessment based on heartbeat dynamics," Scientific reports, vol. 4, 2014.

[16] J. Cui, T. E. Wilson, and C. G. Crandall, "Baroreflex modulation of muscle sympathetic nerve activity during cold pressor test in humans," American Journal of Physiology-Heart and Circulatory Physiology, vol. 282, no. 5, pp. H1717-H1723, 2002.

[17] G. Valenza, L. Citi, E. P. Scilingo, and R. Barbieri, "Point-process nonlinear models with laguerre and volterra expansions: Instantaneous assessment of heartbeat dynamics," IEEE Transactions on Signal Processing, vol. 61, no. 11, pp. 2914-2926, 2013. 